\title{
Molecular Cloning, Sequencing, and Expression of a Fibrinolytic Serine-protease Gene from the Earthworm Lumbricus rubellus
}

\author{
Il Hwan Cho \\ Department of Biological Sciences, Konkuk University, Seoul 143-701, Korea \\ 'Shinpoong Pharmaceutical Co., Moknae-dong, Ansan, Kyunggido 425-100, Korea \\ Lab of Microbial Functions, Korea Research Institute of BioSciences and Biotechnology, Daejon 305-333, Korea
}

Received 18 December 2003, Accepted 25 February 2004

The full-length cDNA of the lumbrokinase fraction 6 (F6) protease gene of Lumbricus rubellus was amplified using an mRNA template, sequenced and expressed in $E$. coli cells. The $\mathrm{F} 6$ protease gene consisted of pro- and mature sequences by gene sequence analysis, and the protease was translated and modified into active mature polypeptide by $\mathrm{N}$-terminal amino acid sequence analysis of the F6 protease. The pro-region of F6 protease consisted of the 44 residues from methionine- 1 to lysine- 44 , and the mature polypeptide sequence (239 amino acid residues and one stop codon; $720 \mathrm{bp}$ ) started from isoleucine-45 and continued to the terminal residue. F6 protease gene clones having pro-mature sequence and mature sequence produced inclusion bodies in $E$. coli cells. When inclusion bodies were orally administrated rats, generated thrombus weight in the rat's venous was reduced by approximately 60\% versus controls. When the inclusion bodies were solubilized in pepsin and/or trypsin solutions, the solubilized enzymes showed hemolytic activity in vitro. It was concluded the $\mathrm{F} 6$ protease has hemolytic activity, and that it is composed of pro- and mature regions.

Keywords: Gene cloning, Lumbricus rubellus, Lumbrokinase, Protease

\section{Introduction}

Fibrinolytic proteases in the earthworm, Lumbricus rubellus have been purified and characterized by several investigators (Park et al., 1989; Mihara et al., 1991, 1993; Nakajima et al., 1993; Jeon et al., 1995; Cho et al., 2004) and found to

\footnotetext{
*To whom correspondence should be addressed.

Tel: 82-2-450-3426; Fax: 82-2-452-9715

E-mail: hhlee@konkuk.ac.kr
}

hydrolyze plasmogen-rich fibrin and plasmogen-free fibrin. Earthworm protease appeared a mixture of six iso-enzyme proteins of molecular weight 25 to $32 \mathrm{kDa}$. Fibrinolytic proteases dissolve blood fibrin clots, and are important chemotherapeutic agents (Mihara et al., 1989; Ryu et al., 1994, 1995; Park et al., 1999).

The sequences of the F-III-2 and F-III-1 fraction isoenzyme genes of $L$. rubellus were reported by Sugimoto and Nakajima (2001), and Sun et al. (2002) and Xu et al. (2002), reported the sequences of the lumbrokinase genes $\mathrm{PI}_{239}$ and $\mathrm{PV}_{242}$ of $L$. bimastus. The other four iso-proteases and differences between the two iso-proteases of $L$. rubellus produced by different strains have not been determined. Thus we undertook to clone and express the protease genes of a Korean L. rubellus strain, which it was hoped would be useful for clinical applications. The aims of this study were; (1) to clone and sequence one of the fibrinolytic protease genes, (2) to determine the levels of expression of the protease gene in $E$. coli, and (3) to examine the fibrinolytic activity of the cloned prokaryotic products.

\section{Materials and Methods}

Animals Earthworms (Lumbricus rubellus) obtained from the Giheung Farmer School, Korea, were used as the lumbrokinase source. A specific pathogen free, eight-week-old Sprague-Dawley male rat strain $(300-350 \mathrm{~g})$, was maintained at $23.1^{\circ} \mathrm{C}$ in $55.5 \%$ humidity with 10-18 aeration changes per hour under a $12 \mathrm{~h}$ light and dark cycle of 300-500 luxes, and used to examine blood clot hydrolysis.

Oligonucleotide primers Nine oligonucleotide primers recognizing the antisense and sense strands of the 5- and 3-ends of the fibrinolytic F6 protease coding sequence were used for PCR (94, $1 \mathrm{~min}$; 60, $2 \mathrm{~min}$; 72, $3 \mathrm{~min}$; 35 cycles) to generate a clonable fragment encompassing only the coding region. These primers were synthesized at Bionics, Seoul, Korea, and are illustrated in Table 1. 
Table 1. List of oligonucleotides

\begin{tabular}{cl}
\hline Primers & \multicolumn{1}{c}{ Sequences $\left(5^{\prime} \rightarrow 3^{\prime}\right)$} \\
\hline AP & 5'-GGCCACGCGTCGACTAGTAC(T) $)_{17} 3^{\prime}$ \\
UAP & 5'-CUACUACUACUAGGCCACGCGTCGACTAGTAC-3' \\
GSP & 5'-ATTGTCGGAGGAATTGAAGCCAGACCATACGAGTTC-3' \\
AFA & 5-GGAGACTTCCAAGGTCTTAGCTATCACTTAAGCAC-5' \\
AFAP & 5'-CTGGTTCGGCCCACCTCTGAAGGTTCCAGAATCGATAG-3' \\
5'RGSP & 5'-TAGATATCATCGCAGAAGGC-3' \\
NCP & 5'-AGGAGGAAACACATATGTTACTTCTCGCCCTTGCA-3' \\
FCP & 5'-GCGCGCCATATGTAAATGTGTCATTCGTTATAA-3' \\
MNCP & 5'-AGGAGGAAACATATGATTGTCGGAGGAATTGAAG-3' \\
\hline
\end{tabular}

Nine oligonucleotide primers that recognized the antisense and sense strands of the 5'- and 3'-ends of the fibrinolytic F6 protease coding sequence were used in a PCR reaction to generate a clonable fragment encompassing only the coding region.

The primers, and AP and UAP sequences, were determined using a 5'-RGSP kit (Clontech, Palo Alto, USA). The AP primer contained oligo-T sequences, Mlu I, Sal I and Spe I sites. The AFA primer was the AmpliFINDER anchor primer, and the AFAP primer was the counterpart of the AFA primer. The GSP primer, which was used for DNA amplification, was designed from the Nterminal amino acid sequences of $\mathrm{F} 6$ protease (Cho et al., 2004). The primer 5'-RGSP, which was used for PCR amplification, was based on the $450 \mathrm{bp}$ upstream sequence of the deduced $\mathrm{N}$-terminal amino acid sequence of the mature region of the F6 protease gene (Fig. 2). The primer (NCP) was prepared using the N-terminal amino acid sequences of the F6 pro-mature sequence (Fig. 2), and contained the Nde I cleavage site (underlined). When this cleavage site was digested with the Nde I, a starting ATG codon was generated. The primer (FCP), the counterpart primer of NCP, was deduced from an area near the stop codon of the F6 mature protease gene and contained a Nde I cleavage site (underlined) (Fig. 2). The primer (MNCP) was deduced from the N-terminal amino acid sequence of the mature region of the F6 protease gene and contained the Nde I site (underlined) (Fig. 2).

Molecular cloning of the LKF6 protein gene The full-length fibrinolytic F6 protease gene was constructed by combining two cDNA fragments of $0.9 \mathrm{~kb}$, and $0.4 \mathrm{~kb}$, which were synthesized from mRNA using the procedures for 3'- or 5'-rapid amplification of cDNA ends and PCR (Fig. 1). A recombinant plasmid was constructed using standard protocols (Sambrook et al., 1989; Lee $e t$ al., 1998a, 1998b; Uh et al., 2001).

Construction of the p3LK6 clone One gram of L. rubellus was crushed and homogenized for $20 \mathrm{sec}$ in $10 \mathrm{ml}$ of guanidium thiocyanate buffer (4 M guanidium thiocyanate, $0.1 \mathrm{M}$ Tris- $\mathrm{HCl}$.

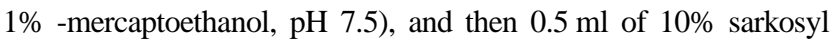
(Sigma, St. Louis, USA) was added. This solution was mixed

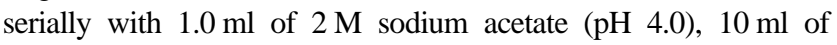
phenol and $2 \mathrm{ml}$ of chloroform/isoamyl alcohol (49:1) and then centrifuged for $20 \mathrm{~min}$ at $4^{\circ} \mathrm{C}$ and $10,000 \times g$. The supernatants were collected, $10 \mathrm{ml}$ of isopropanol added, and the solution was stored for $1 \mathrm{~h}$ to allow precipitation. The mRNA precipitates were then harvested by centrifugation for $20 \mathrm{~min}$ at $4^{\circ} \mathrm{C}$ and $10,000 \times g$, and the pellets were washed with $80 \%$ ethanol twice and dried;
$1.3 \mathrm{mg}$ of total RNA was obtained from the L. rubellus lysates. Six micrograms of the mRNA were purified from $300 \mu \mathrm{g}$ of the total RNA using oligo (dT) 25 Beads (Novagen, Milwaukee, USA). First strand cDNAs were synthesized using mRNA templates and a 3RACE kit (Gibco, Gaithersburg, USA) (Fig. 1). One $\mu \mathrm{g}$ of the mRNAs and 1.0 pmol of adapter primer (AP) were suspended in $13 \mu \mathrm{l}$ of $\mathrm{H}_{2} \mathrm{O}$ and heated for $3 \mathrm{~min}$ at $80^{\circ} \mathrm{C}$. Then $2.0 \mu \mathrm{l}$ of $10 \mathrm{X}$ cDNA synthesis buffer [1.0 $\mu \mathrm{l}$ of $10 \mathrm{mM}$ dNTPs, $2.0 \mu \mathrm{l}$ of $0.1 \mathrm{M}$ DTT, and $1.0 \mu \mathrm{l}$ of $10 \mathrm{units} / \mu \mathrm{l}$ superscript RT (reverse transcriptase)] were added and the resultant mixture was heated for $30 \mathrm{~min}$ at $42^{\circ} \mathrm{C}$. After heating, $1.0 \mu \mathrm{l}$ of (1000 units/ml) RNaseH was added to the solution, which was reheated for $10 \mathrm{~min}$ to remove the mRNAs. The first cDNA templates were amplified by PCR with primers AP and UAP, and a vent DNA polymerase (NEB, Beverly, USA). PCR was done over 35 cycles of denaturation for $1 \mathrm{~min}$ at $94^{\circ} \mathrm{C}$, annealing for $2 \mathrm{~min}$ at $60^{\circ} \mathrm{C}$, and extension for $3 \mathrm{~min}$ at $72^{\circ} \mathrm{C}$. After PCR, the resulting products were run in $1.0 \%$ agarose gels, and the band of interest was eluted, purified, and extracted with a QiaEX kit (Qiagen, Hilden, Germany). The purified DNA fragment was named LK6N cDNAs $(0.9 \mathrm{~kb})$. The LK6N DNA fragment $(100 \mathrm{ng})$ was the blunt-ligated into pT7 Blue vector (50 ng) using 2 units of T4 ligase (Novagen), and the resulting recombinant, which was named p3LK6 plasmid, was transformed into E. coli DH5 $\alpha$ (Sambrook et al., 1989).

Construction of p5LK6 clone First strand cDNA was synthesized using the mRNA templates of L. rubellus and a 5'-RACE kit (Clontech, Palo Alto, USA) with the primers AFA and AFAP (Fig. 1). The cDNA fragments were amplified using PCR as in the above-mentioned procedure, and the resultant double stranded cDNA was named LK6C cDNA $(0.4 \mathrm{~kb})$. LK6C was cloned into pT7 Blue vector and the resulting clone was named p5LK6, and transformed into E. coli DH5 $\alpha$.

Construction of pLK6T clone p3LK6 was digested with Nco I and Sac I enzymes, and the resultant Nco I/Sac I fragment was cloned into the Nco I and Sac I sites of the p5LK6 clone. The resulting plasmid was called pLK6T recombinant (Fig. 1).

Expression construct, pLK-prom clone Using the pLK6T recombinant, the pro-mature gene sequences of the LKF6 gene were 


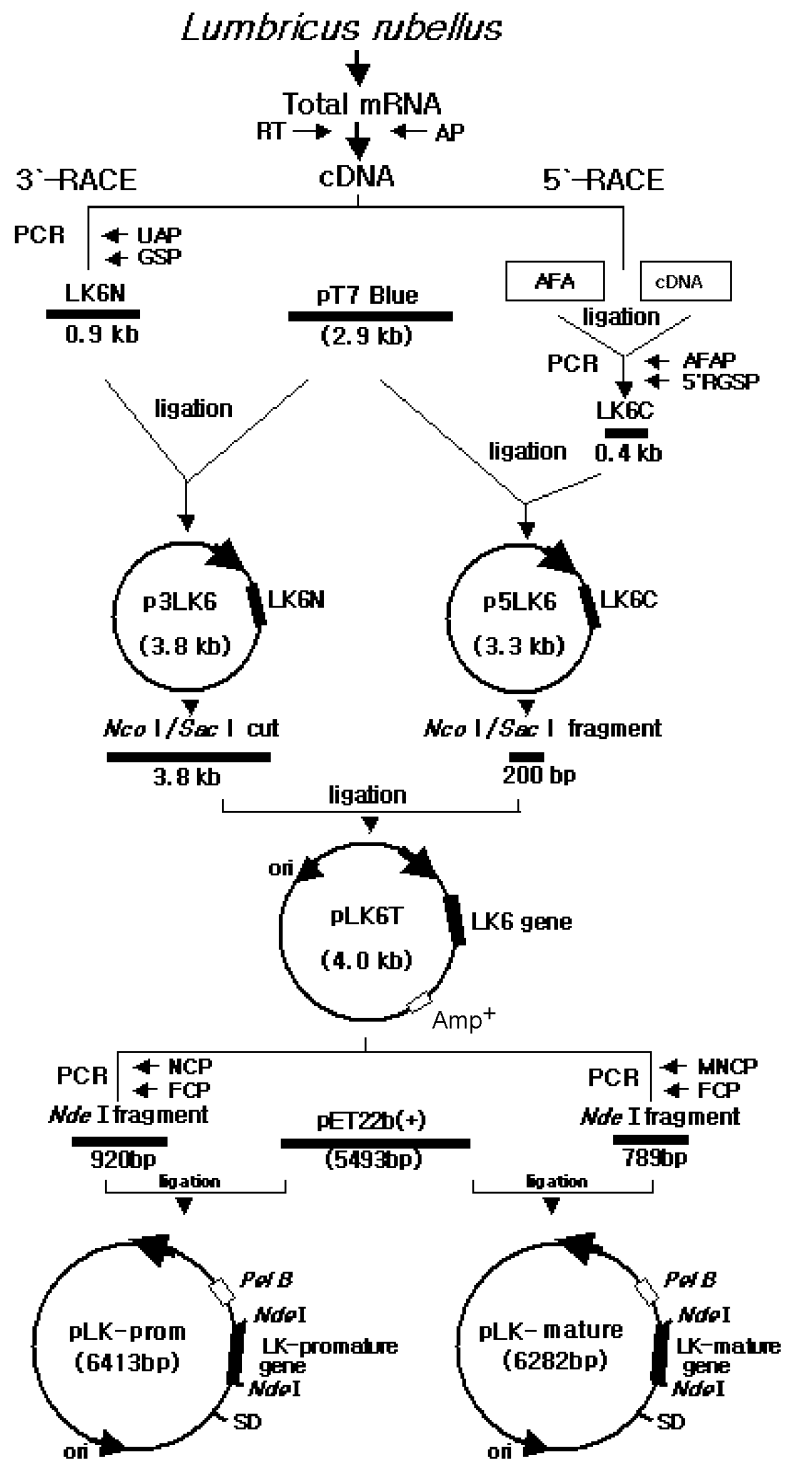

Fig. 1. Construction of pLK6T, pLK-prom and pLK-mature recombinant plasmids containing the F6 gene. Using mRNA templates of the F6 gene in $L$. rubellus lysates, the cDNAs were synthesized with 3- or 5-rapid amplification of cDNA end kits, primers (AP, UAP, GSP, AFA, in Table 1) and a PCR. The cDNAs were cloned into pT7 plasmids, and the full-length cDNAs of the F6 gene clone were generated by combining the Nco I and Sac I 200 bp DNA fragments in p5LK plasmids into the same sites of the p3LK6. The resulting clone was called the pLK6T recombinant. Two expression clones, containing the sequences of the pro-mature or mature regions, were synthesized and cloned into $\mathrm{pET} 22 \mathrm{~b}(+)$ vector using primers containing Nde I sites. PCR was then performed on the resulting recombinant plasmids, which were named pLK-prom and pLK-mature.

amplified by PCR using the primers NCP and FCP containing Nde I sites. Eight base pairs of space were placed between the ShineDalgarno sequence (SD) and the ATG codon in the pET22b(+) vector. The PCR product was digested with $N d e$ I and cloned into the Nde I site of pET22b(+) vector, called pLK-prom recombinant plasmid (6.413 kb) (Fig. 1), and then transformed into the E. coli.

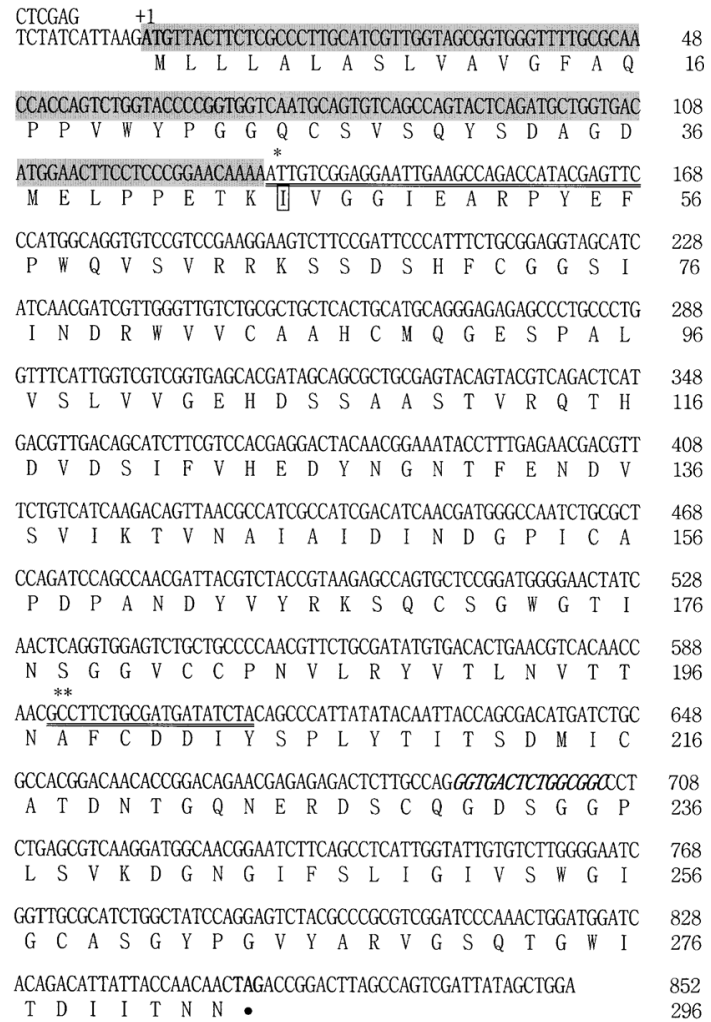

Fig. 2. Analysis of the nucleotide sequences of the ORF of the F6 protease gene and its deduced amino acid sequence (GenBank accession No. LK-6 AF304199). The sequences of the proand of the mature region of the F6 protease gene are given. The gene sequences were determined using an automatic DNA sequencer. The 44 pro-region amino acids are shaded. The sequence of the mature protein starts from the 45-isoleucine through to the terminal residue. The $\mathrm{N}$-terminal amino acid sequences of the F6 mature protein are underlined. Italic bases represent conserved residues around a/the serine protease active site. The initiation codon is boldfaced, and represents the termination codon. *, sequence of primer GSP. **, sequence of primer 5RGSP.

Expression construct, pLK-mature clone Using the pLK6T recombinant, the mature gene sequence of the LKF6 gene was amplified by PCR with FCP and MNCP primers containing Nde I sites. The resulting PCR product was cloned into the Nde I site of pET22b(+) vector and called pLK-mature recombinant plasmid $(6.282 \mathrm{~kb})$ (Fig. 1), which was transformed into E. coli.

Sequencing and computer analysis of LK6 gene cDNAs The LK6N cDNA fragment in the p3LK6 clone, LK6C cDNA fragment in the p5LK6 clone, and LK6 gene DNA in the pLK6T clone were purified through Qiagen tip to determine their DNA sequences.

Initially, an Exo/Mung Bean unidirectional deletion kit (Qiagen) was used to obtain deletion fragments. These DNA fragments were amplified using a PRISM Ready Reaction dye primer cycle sequencing kit (Qiagen). The sequences of the amplified cDNA fragments were determined using an automatic DNA sequencer (Bio-Rad Lab, Richmond, USA) (Crothers and Dreak, 1992). DNA fragments were sequenced on both strands, and the LK6N, LK6C 
and LK6 cDNA sequences were fully determined. The whole amino acid sequence of the LKF6 protein was deduced from the complete LK6 cDNA sequence. The DNA base sequences of all fragments were analyzed using the MacDNASIS program (Apple Computer Ltd., Cupertino, USA).

Preparation of fibrinolytic protease from $E$. coli $E$. coli cells containing the recombinant plasmids, pLK-prom or pLK-mature (Fig. 2), were cultured overnight on LB agar plate containing ampicillin $(100 \mu \mathrm{g} / \mathrm{ml})$, and a single colony was then transferred into $20 \mathrm{ml}$ of $\mathrm{LB}$ broth and allowed to grow overnight at $37^{\circ} \mathrm{C}$ with shaking. The seed cultures obtained were then inoculated into 500 $\mathrm{ml}$ of LB broth containing ampicillin $(100 \mu \mathrm{g} / \mathrm{ml})$ and allowed to grow at $37^{\circ} \mathrm{C}$ until reaching an optical density of 1.2 at $600 \mathrm{~nm}$. At this point, $1.0 \mathrm{mM}$ of IPTG (isopropyl- $\alpha$-thio-galactopyranoside) (Stratagene, Heidelbergh, Germany) was added, and the cells were re-incubated for $4 \mathrm{hrs}$ at $37^{\circ} \mathrm{C}$, and centrifuged at $12,000 \times g$ for 30 $\min$ at $4^{\circ} \mathrm{C}$. The insoluble protein pellets and supernatants were then separated, and the supernatants were concentrated with $1.0 \%$ of trichloroacetic acid and freeze-dried. The insoluble protein pellets were sonicated and centrifuged at $12,000 \times g$ for $30 \mathrm{~min}$ at $4^{\circ} \mathrm{C}$. The insoluble protein pellets (LK inclusion bodies) were washed twice with $1 \%$ Triton X-100, resuspended in $150 \mu \mathrm{l}$ of $1.0 \mathrm{mM}$ Tris $\cdot \mathrm{HCl}$ buffer (pH 7.6), and used for SDS-PAGE (Laemmli, 1970) and to determine fibrinolytic activity (Kumada et al., 1980; Cho et al., 2004).

In vitro fibrinolytic activity of the protease from $\boldsymbol{E}$. coli To estimate the in vitro activity of the inclusion bodies, $1.0 \mathrm{mg}$ of the inclusion bodies of lumbrokinase produced in E. coli cells was suspended in $12 \mathrm{ml}$ of $0.05 \mathrm{M} \mathrm{HCl}-\mathrm{KCl}$ buffer, $\mathrm{pH} \mathrm{2.0.} \mathrm{The}$ suspension was then ultrasonicated for $10 \mathrm{~min}$ at $37^{\circ} \mathrm{C}$ and $100 \mu \mathrm{l}$ of pepsin solution ( 500 units $/ \mathrm{ml}$ ) was added. The mixture was then incubated in a shaking water bath at $37^{\circ} \mathrm{C}$ for $30 \mathrm{~min}$. To create an intestinal environment, the $\mathrm{pH}$ of the mixture was neutralized by adding $1.0 \mathrm{~N} \mathrm{NaOH}$. Trypsin solution (500 units/ml) was then added to the neutralized mixture, and incubated in a shaking water bath at $37^{\circ} \mathrm{C}$ for $60 \mathrm{~min}$. Then $10 \mu \mathrm{l}$ of the solubilized enzyme solution was plated on the fibrin plate (Cho et al., 2004), incubated for $15 \mathrm{~h}$ at $37^{\circ} \mathrm{C}$, and the hydrolyzed clear zone was measured (Cho et al., 2004).

In vivo fibrinolytic activity of the protease from $\boldsymbol{E}$. coli The fibrinolytic activity of the lumbrokinase (LK) inclusion bodies produced by the $E$. coli system, was evaluated using a rat model of venous thrombosis (Kumada et al., 1980) with some modification. Briefly, the inclusion bodies were resuspended in $50 \mathrm{mM}$ phosphate buffer ( $\mathrm{pH} 7.4$ ), washed twice with $1.0 \%$ Triton X-100, rewashed with phosphate buffer, and freeze-dried. Eight rats were anaesthetized with ketamine (Yuhan Pharmaceutical Co, Seoul, Korea) and a midline incision was made in the abdomen. The inferior vena cava was exposed and a stainless steel wire coil was inserted $15 \mathrm{~mm}$ into the lumen of the inferior vena cava at the left renal vein branching. The inclusion bodies were then suspended for two hours in a $0.5 \%$ carboxylmethyl cellulose solution, after which, the inclusion body solution (at a dose of $50 \mathrm{mg} / 10 \mathrm{ml} / \mathrm{kg}$ body wt/ d) was administered orally for $5 \mathrm{~d}$. The control group of eight rats received only $10 \mathrm{ml}$ of $0.5 \%$ carboxylmethyl cellulose solution for
$5 \mathrm{~d}$. One hour after the final administration, the rats were laparotomized under ketamine anesthesia. Immediately after clamping the vena cava, the wire, together with its thrombus, was removed carefully. The weight of the thrombus on the steel wire was determined as total protein.

\section{Results and Discussion}

Cloning and sequence analyses of the F6 protease gene The full-length of the F6 protease gene was cloned by combining two cDNA fragments, and the resulting clone was named pLK6T recombinant plasmid (Fig. 1). The F6 protease gene in the pLK6T plasmid was sequenced using an automatic DNA sequencer (ABI Prism 310) (Perkin Elmer, Foster, USA), according to the manufactures directions. The open reading frame (ORF) sequence of the F6 protease gene and its deduced amino acid residues (GenBank No. LK-6 AF304199) are illustrated in Fig. 2. The ORF sequence contains 284 codons ( $852 \mathrm{bp}$ ), which encode a polypeptide of 283 amino acid residues. These results were consistent with a report (AF433650) on L. bimastus (Sun et al., 2002) (Fig. 3). Sugimoto and Nakajima (2001) reported only the mature protein sequence of the ORF of $L$. rubellus strain, and found that the LK gene sequence is highly conserved in the genus Lumbricus. The differences in the LK gene and protein sequences in reported L. bimastus (Sun et al., 2002) and $L$. rubellus strains (Sugimoto and Nakajima, 2001) are shown in Fig. 3 and 4. When our data is compared our data with the previously reported LK gene sequence (AF433650) of $L$. bimastus by Sun et al. (2002), eight nucleotide differences were found, which corresponded two codon were changes in the coding region of 852 base pairs (Fig. 3 and 4). However, compared our data with the previously reported mature sequence of the LK fraction of the F-III-1 gene (AB045720) of L. rubellus by Sugimoto and Nakajima (2001), 76 nucleotide differences $(10.5 \%)$ were found, corresponding to which 20 codon changes in the mature coding region of 720 base pairs (Fig. 3 and 4). Sugimoto and Nakajima (2002) did not report the pro-region sequence of the LK gene. In addition, when the mature protein gene sequence of $L$. rubellus reported by Sugimoto and Nakajima (2001) was compared with the previously reported LK gene sequence of L. bimastus by Sun et al. (2002), 73 nucleotide differences $(10.1 \%)$ were found, corresponding to 19 codon changes in the mature region of 720 base pairs (Fig. 3 and 4). Also, in the mature protein region (720 base pair) reported by Sugimoto and Nakajima one codon (AGC) between nucleotide 612 and nucleotide 616 was deleted (Fig. 3). In the report by Sun et al. (2002) and according to our data one codon (AGC) between nucleotide 612 and 616 was inserted and one amino acid residue (serine) at the amino acid residue-205 added when compared with Sugimoto and Nakajima's report (Fig. 3 and 4). These results indicate that the LK gene sequence of $L$. rubellus strains is less conserved than L. bimastus strain. The termination codon was TAG according to our data (Fig. 2), but 


\begin{abstract}
AF304199 1 ATGTTACTTCTCGCCCTTGCATCGTTGGTAGCGGTGGGTTTTGCGCAACCACCAGTCTGGTACCCCGGTGGTCAATGCAg AF433650 1 ATGTTACTTCTCGCTCTTGCATCGTTGGTAGCGGTGGGCTTTGCGCAACCACCAGTCTGGTACCCCGGTGGTCAATGCGG

AF304199 81 TGTCAGCCAGTACTCAGATGCTGGTGACATGGAACTTCCTCCCGGAACAAAAATTGTCGGAGGAATTGAAGCCAGACCAT AF433650 81 TGTCAGCCAGTACTCAGATGCTGGTGACATGGAACTTCCTCCCGGAACAAAAATTGTCGGAGGAATTGAAGCCAGACCAT AB 045720 1

AF304199 161 ACGAGTTCCCATGGCAGGTGTCCGTCCGAAGGAAGTCTTCCGATTCCCATTTCTGCGGAGGTAGCATCATCAACGATCGT AF433650 161 ACGAGTTCCCATGGCAGGTGTCCGTCCGAAGGAAGTCTTCCGATTCCCATTTCTGCGGAGGTAGCATCATCAACGATCGT AB045720 53 ACGAGTTCCCATGGCAGGTGTCCGTCCGAAGGAAGTCTTCCGATTCCCATTTCTGCGGAGGTAGCATCATCAACGATCGT

AF304199 241 TGGGTTGTCTGCGCTGCTCACTGCATGCAGGGAGAGAGCCCTGCCCTGGTTTCATTGGTCGTCGGTGAGCACGATAGCAG AF433650 241 TGGGTTGTCTGCGCTGCTCACTGCATGCAGGGAGAGAGCCCTGCCCTGGTTTCATTGGTCGTCGGTGAGCACGATAGCAG AB0 45720133 TGGGTTGTCTGCGCTGCTCACTGCATGCAGGGAGAGGCCCCCGCTCTGGTTTCATTGGTCGTGGGTGAGCACGACAGGAG

AF304199 321 CGCTGCGAGTACAGTACGTCAGACTCATGACGTTGACAGCATCTTCGTCCACGAGGACTACAACGGAAATACCTTTGAGA AF433650 321 CGCTGCGAGTACAGTACGTCAGACTCATGACGTTGACAGCATCTTCGTCCACGAGGACTACAACGGAAATACCTTTGAGA AB0 45720213 TGCAGCGATACAGTACGTCAGACTCATGACGTTGATAGCATCTTCGTCCACGAGGACTACAACGCAAATACCCTAGAGA

AF304199 401 ACGACGTTTCTGTCATCAAGACAGTTAACGCCATCGCCATCGACATCAACGATGGGCCAATCTGCGCTCCAGATCCAGCC AF433650 401 ACGACGTTTCTGTCATCAAGACAGTTAACGCCATCGCCATCGACATCAACGTTGGGCCAATCTGCGCTCCAGATCCAGCC AB0 45720293 ACGACGTTTCTGTCATCAAGACATCTGTTGCCATCACTTTCGACATCAACGTTGGTCCAATCTGCGCCCCAGATCCGGCT

AF304199 481 AACGATTACGTCTACCGTAAGAGCCAGTGCTCCGGATGGGGAACTATCAACTCAGGTGGAGTCTGCTGCCCCAACGTTCT AF433650 481 AACGATTACGTCTACCGTAAGAGCCAGTGCTCCGGATGGGGAACTATCAACTCAGGTGGAGTCTGCTGCCCCAACGTTCT AB0 45720373 AACGACTACGTCTACCGTAAGAGCCAGTGTTCCGGATGGGGAACTATCAATTCAGGTGGAATCTGCTGTCCCAACGTTCT

AF304199 561 GCGATATGTGACACTGAACGTCACAACCAACGCCTTCTGCGATGATATCTACAGCCCATTATATACAATTACCAGCGACA AF433650 561 GCGATATGTGACACTGAACGTCACAACCAACGCCTTCTGCGATGATATCTACAGCCCATTATATACAATTACCAGCGACA AB0 45720453 GCGATACGTGACGCTGAATGTACAACCAACCAATTCTGCGAAGATGTATAC---CCACTAAATTCAATCTACGACGATA

AF304199 641 TGATCTGCGCCACGGACAACACCGGACAGAACGAGAGAGACTCTTGCCAGGGTGACTCTGGCGGCCCTCTGAGCGTCAAG AF433650 641 TGATCTGCGCCACGGACAACACCGGACAGAACGAGAGAGACTCTTGCCAGGGTGACTCTGGCGGCCCTCTGAGCGTCAAG AB0 45720530 TGATTTCGCGTCGGACAACACTGGGGGTAACGACAGAGACTCCTGCCAGGGTGACTCCGGCGGCCCTCTGAGCGTCAAG

AF304199 721 GATGGCAGCGGAATCTTCAGCCTCATTGGTATTGTGTCTTGGGGAATCGGTTGCGCATCTGGCTATCCAGGAGTCTACGC AF 433650721 GATGGCAGCGGAATCTTCAGCCTCATTGGTATTGTGTCTTGGGGAATCGGTTGCGCATCTGGATATCCAGGAGTCTACGC AB0 45720610 GATGGCAGTGGAATCTTCAGCCTGATTGGTATTGTGTCTTGGGGAATITGTTGCGCTTCTGGCTATCCAGGAGTCTACTC

AF304199 801 CCGCGTCGGATCCCAAACTGGATGGATCACAGACATTATTACCAACAACTAG

AF433650 801 CCGCGTCGGGTCCCAAACTGGATGGATCACAGACATCATCACCAACAACTAA

AB0 45720690 CCGCGTCGGATTCCATGCTGCATGGATCACCGACATCATCACCAACAACTAA
\end{abstract}

Fig. 3. Comparison of the LK gene sequences of L. rubellus and L. bimastus strains. The base sequence with GenBank accession number AF304199 is the LK gene of L. rubellus isolated in Korea, the sequence AF433650 (Sun et al., 2002) is the LK gene of $L$. bimastus strain, and the sequence AB045720 (Sugimoto and Nakajima, 2001) is LK gene of a L. rubellus strain isolated in Japan. Black bars with white text indicate different bases. The numbers on the left indicate the nucleotide numbers. (codon: ---) between nucleotides 612 and 616 of AB045720 means a deleted codon versus the other two sequences.

TAA according to Sugimoto and Nakajima (2001) and Sun $e t$ al. (2002).

The F6 protein gene might consist of duplicate regions of the full-length amino acid sequence of the F6 protein, based on the N-terminal amino acid sequence (Cho et al., 2004). The active form (the mature protein) of F6 polypeptides is initiated from isoleucine-45 and not methionine. This means that the polypeptides produced may be modified after translation. The F6 gene has a pro-region sequence upstream from its mature sequence. The amino acid sequence of the pro-region (44 amino acid residues) in the F6 polypeptide starts at methionine- 1 and continues to lysine-44 (shaded box). The active mature polypeptide sequence (239 amino acid residues and one stop codon; $720 \mathrm{bp}$ ) starts from isoleucine-45 to the terminal residue. Thus the pro-region consists of $132 \mathrm{bp}$ (44 amino acid residues), and the mature protein sequence of the F6 protein gene is of $720 \mathrm{bp}$, encoding a polypeptide of 239 amino acid residues. The F6 polypeptide enzymes have a potential $N$-glycosylation site at amino acid residues 149-152 and a serine protease active site at 231-235amino acid residues. The deduced sequence of the amino acid residues in the mature polypeptide contains a high percentage (11-14\%) of asparagine and aspartic acid, and the percentage of lysine was lower than former amino acid.

Fibrinolytic activity of F6 proteases produced by $\boldsymbol{E}$. coli clones The pLK-prom and the pLK-mature clones were constructed according to the scheme shown in Fig. 1. The two clones were transformed in E. coli system, and produced inclusion body types of F6 proteases, were identified by SDSPAGE (Fig. 5 lanes 2 and 3). Lane 2 is the protein produced by the pLK-mature clone, which has a molecular weight of 


\begin{abstract}
AF304199
1 MLLLALASLVAVGFAQPPVWYPGGQC

AB0 45720 VSQYSDAGDMELPPGTKIVGGIEARPYEFPWOVSVRRKSSDSHFCGGSIINDR

AF304199 81 WVVCAAHCMQGESPALVSLVVGEHDSSAASTVRQTHDVDSIFVHEDYNGNTFENDVSVIKTVNAIAIDINDGPICAPDPA

AF433650 81 WVVCAAHCMQGESPALVSLVVGEHDSSAASTVRQTHDVDSIFVHEDYNGNTEENDVSVIKTVNAIAIDINVGPICAPDPA

AB0 $45720 \quad 45$ WVVCAAHCMQGEAPALVSLVVGEHDRSAASTVRQTHDVDSIFVHEDYNANTLENDVSVIKTSVAITEDINVGPICAPDPA

AF304199 161 NDYVYRKSQCSGWGTINSGGVCCPNVLRYVTLNVTTNAFCDDIYSPLYTITSDMICATDNTGQNERDSCQGDSGGPLSVK AF433650 161 NDYVYRKSQCSGWGTINSGGVCCPNVLRYVTLNVTTNAFCDDIYSPLYTITSDMICATDNTGONERDSCOGDSGGPLSVK AB0 45720125 NDYVYRKSQCSGWGTINSGGICCPNVLRYVTLNVTTNEFCEDVY-PLNSIYDDMICASDNTGGNDRDSCQGDSGGPLSVK
\end{abstract}

AF304199 241 DGSGIFSLIGIVSWGIGCASGYPGVYARVGSOTGWITDIITNN

AF433650 241 DGSGIFSLIGIVSWGIGCASGYPGVYARVGSOTGWITDIITNN

AB045720 204 DGSGIFSLIGIVSWGIGCASGYPGVYSRVGFHAAWITDIITNN

Fig. 4. Comparison of the LK protein sequences of $L$. rubellus and L. bimastus strains. The amino acid sequence with GenBank accession number AF304199 is the LK protein of L. rubellus isolated in Korea, the sequence AF433650 is the LK protein of $L$. bimastus strain, and the sequence AB045720 is LK protein of L. rubellus strain isolated in Japan. Black bars with white text indicate different amino acids. The numbers on the left indicate amino acid residues. (-) at amino acid residues-245 on AB045720 indicates a deleted amino acid versus the other two sequences.

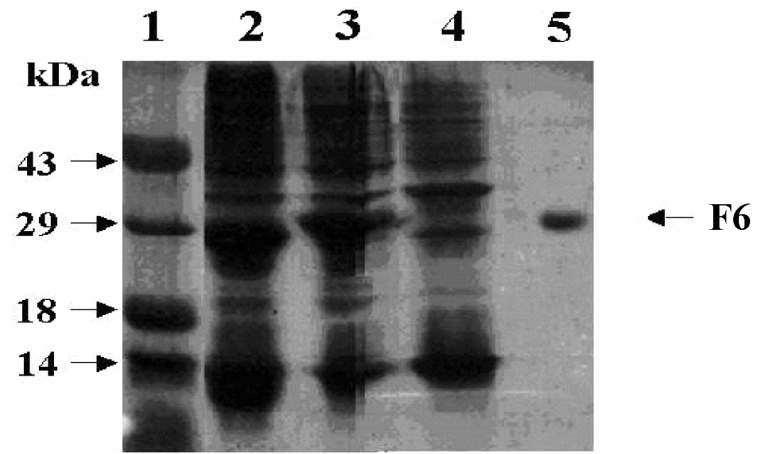

Fig. 5. SDS-PAGE patterns of F6 inclusion bodies produced by clones. E. coli containing the clones (pLK-prom and pLKmature) produced inclusion bodies of F6 protein. The F6 inclusion bodies were dissolved in the $50 \mathrm{mM}$ phosphate buffer and run on $12 \%$ SDS-PAGE. The lanes shown are: 1, molecular weight maker; 2 , proteins produced by pLK-mature clone; 3 , proteins produced by pLK-prom clone; 4 , proteins produced by pET22b vector (control), and 5, the F6 fraction protein. Numbers on the left correspond to the positions of the molecular weight markers (in $\mathrm{kDa}$ ) (ovalbumin $43 \mathrm{kDa}$, carbonic anhydrase 29 $\mathrm{kDa}$, b-lactogloblin $18 \mathrm{kDa}$, and lysozyme $14 \mathrm{kDa}$ ). F6 indicates F6 protein.

approximately $28 \mathrm{kDa}$, and lane 3 is the pro-mature protein produced by the pLK-prom clone, which has a molecular weight of approximately $31 \mathrm{kDa}$.

Lane 4 shows the proteins produced by the $E$. coli control, and lane 5 the protein from fraction 6 , which has a molecular weight of approximately $33 \mathrm{kDa}$. The mature and pro-mature proteins showed lower molecular weights than the proteases from fraction 6 . This means that the mature and promature proteins were not modified in E. coli cells. These results indicate that the two clones produced different molecular weights gene products due to the different lengths of their genes.

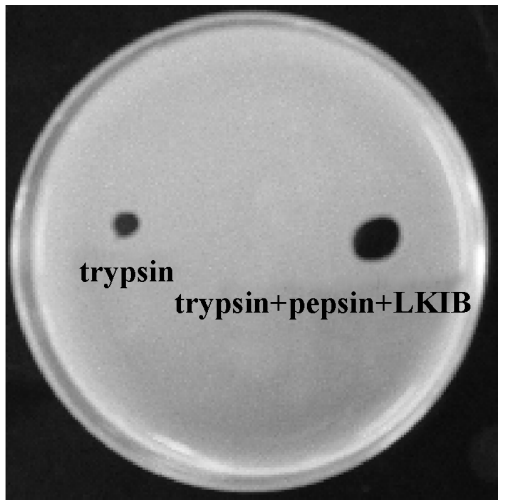

Fig. 6. In vitro fibrinolytic activity on fibrin plates of the LK inclusion bodies from E. coli. A 5\% fibrinogen solution was prepared using a fibrin plate buffer. $10 \mathrm{ml}$ of the mixture was distributed into sterile Petri-dishes, and then $50 \mu \mathrm{l}$ of $20 \mathrm{NIH}$ units $/ \mathrm{ml}$ of thrombin solution were slowly mixed into each dish and allowed to solidify for $1 \mathrm{~h}$ at room temperature. $10 \mu \mathrm{l}$ of each LK inclusion body (IB) solution treated with trypsin and/or pepsin was dropped on the fibrin plates and incubated for $15 \mathrm{~h}$ at $37^{\circ} \mathrm{C}$. After incubation, the mean diameters of the hydrolyzed clear zones were measured. Trypsin, IB treated with trypsin; trypsin + pepsin + IB spot, IB treated with trypsin and pepsin.

The inclusion bodies, which were solubilized in pepsin and/ or trypsin solutions (described in "Materials and Methods") showed high hemolytic activity in vitro (Table 2 and Fig. 6). The IB solutions treated with pepsin and trypsin together showed much stronger hemolytic activity than those treated with only trypsin. The IB solution alone was inactive. The inclusion bodies suspended in a $0.5 \%$ carboxylmethyl cellulose solution were administered orally for 5 days. After last administration, the steel wires and attached thrombus were removed, and the thrombus weights determined (described in the Method). The F6 proteases hydrolyzed 62- 
Table 2. In vitro fibrinolytic activity of inclusion bodies

\begin{tabular}{lc}
\hline Biochemical reactions & Diameter of clear zone $(\mathrm{mm})$ \\
\hline LK-IB & $\mathrm{ND}$ \\
pepsin & $\mathrm{ND}$ \\
LK-IB + trypsin & $5.7 \pm 0.4$ \\
LK-IB + pepsin & $\mathrm{ND}$ \\
LK-IB + pepsin + trypsin & $11.2 \pm 1.6^{*}$ \\
\hline
\end{tabular}

LK-IB, the inclusion body of lumbrokinase F6 produced in $E$. coli cells; ND, not detected; *, $p<0.05$ vs trypsin. Data were represented mean $\pm S D(n=5)$.

Table 3. Thrombus weight change after oral administration of the LK inclusion bodies produced in E. coli

\begin{tabular}{llc}
\hline LK IB sources & Treated dose & $\begin{array}{c}\text { Thrombus weight } \\
(\mu \mathrm{g}) \pm \text { S.D }\end{array}$ \\
\hline Control & $\mathrm{CMC} 10 \mathrm{ml} / \mathrm{kg} / \mathrm{d}$ & $1726 \pm 852$ \\
pLK-prom & $50 \mathrm{mg} / \mathrm{kg} / \mathrm{d}$ & $659 \pm 528$ \\
pLK-mature & $50 \mathrm{mg} / \mathrm{kg} / \mathrm{d}$ & $610 \pm 429$ \\
\hline
\end{tabular}

Fibrinolytic activity of the LK inclusion bodies (IB) was evaluated using a rat model of venous thrombosis (Kumada et al., 1980). The inclusion bodies suspended in a $0.5 \%$ carboxylmethyl cellulose (CMC) solution was administered orally for 5 days. One hour after the last administration, the rats were laparotomized. Immediately after clamping the vena cava, the wire with its thrombus was removed carefully. The weight of the thrombus on the steel wire was measured as total protein content.

$65 \%$ of the blood clots on the stainless wire coils in rats when fed orally (Table 3 ). The thrombus weights were reduced to $659(62 \%)$ by the pro-mature enzymes produced by the pLKprom clone and to $610(65 \%)$ by the mature form produced by the pLK-mature clone versus the control group (Table 3 ). The pro-mature and mature proteases produced in the E. coli system had similar fibrinolytic activities in vivo and in vitro, which means that the pro-mature and the mature regions of the F6 proteases may be involved in proteolytic activity. We assumed that when the inclusion body solution was fed to rats that were converted in the intestine to active forms. Further studies are necessary.

We conclude that the F6 proteases consist of pro- and mature regions. The ORF sequence of the pro-mature protease was composed of $852 \mathrm{bp}$ and the mature region was of 720 bp. The mature and pro-mature enzymes produced by $E$. coli were produced as inclusion bodies and showed high fibrinolytic activity in vivo and in vitro.

Acknowledgments This work was supported by a Korea Research Foundation Grant (KRF-2000-015-DP0312).

\section{References}

Cho, I. H., Choi, E. S., Lim, H. G. and Lee, H. H. (2004)
Purification and characterization of six fibrinolytic serineproteases from earthworm Lumbricus rubellus. J. Biochem. Mol. Biol. 37, 199-205.

Crothers, D. M. and Drak, J. (1992) Global features of DNA structure by comparative gel electrophoresis. Methods in Enzymology 212, 46-71.

Jeon, O. H., Moon, W. J. and Kim, D. S. (1995) An anticoagulant/fibrinolytic protease from Lumbricus rubellus. $J$. Biochem. Mol. Biol. 28, 138-1452.

Kumada, T., Ishihara, M., Ogawa, H. and Abiko, Y. (1980) Experimental model of venous thrombosis in rats and effect of some agents. Thrombosis Res. 18, 189-203.

Laemmli, U. K. (1970) Cleavage of structural proteins during the assembly of the head of bacteriophage T4. Nature 227, 680685.

Lee, H. H., Kang, B. J. and Park, K. J. (1998a) Construction of a Baculovirus expression system using $H$. cunea nuclear polyhedrosis virus for eukaryotic cell. J. Microbiol. Biotechnol. 8, 676-684.

Lee, H. H., Moon, E. S., Lee, S. T., Hwang, S. H., Cha, S. C. and Yoo, K. H. (1998b) Construction of a Baculovirus Hyphantria cunea NPV insecticide containing the insecticidal protein gene of Bacillus thuringiensis subsp. kurstaki HD1. J. Microbiol. Biotechnol. 8, 685-691.

Mihara, H., Sumi, H., Yoneta, T., Mizumoto, H., Ikeda, R., Seikl, M. and Maruyama, M. (1991) A novel fibrinolytic enzyme extracted from the earthworm, Lumbricus rubellus. Jpn. J. Phgysiol. 41, 461-472.

Mihara, H., Yineta, T., Sumi, H., Soeda, M. and Maruyama, M. (1989) A possibility of earth worm powder as therapeutic agent for thrombosis. Thromb. Haemosta. 62, 545-549.

Nakajima, N., Mihara, H. and Sumi, H. (1993) Characterization of potent fibrinolytic enzymes in Earthworm, Lumbricus rubellus. Biosci. Biotech. Biochem. 57, 1726-1730.

Park, S. Y., Kye, K. C., Lee, M. H., Sumi, H. and Mihara, H. (1989) Fibrinolytic activity of the earth worm extract. Thromb. Haemosta. 62, 545-550.

Park, Y., Ryu, E., Kim, H., Jeong, J., Kim, J., Shim, J., Jeon, S., Jo, Y., Kim, W. and Min, B. (1999) Characterization of antithrombotic activity of lumbrokinase-immobilized Polyurethane valves in the total artificial heart. Artif. Organs. 23, 210-214.

Ryu, G. H., Han, D. K., Park, S. Y., Kim, M., Kim, Y. H. and Min, B. G. (1995) Surface characteristics and properties of lumbrokinase-immobilized polyurethane. J. Biomed. Mater. Res. 29, 403-409.

Ryu, G. H., Park, S., Kim, M., Han, D. K., Kim, Y. H. and Min, B. G. (1994) Antithrombogenicity of lumbrokinase immobilized polyurethane. J. Biomed. Mater. Res. 28, 10691077.

Sambrook, J., Fritsch, E. F. and Maniatis, T. (1989) Molecular Cloning. A Laboratory Manual, Cold Spring Harbor Laboratory, Cold Spring Harbor, USA.

Sugimoto, M. and Nakajima, N. (2001) Molecular cloning, sequencing, and expression of cDNA encoding serine protease with fibrinolytic activity from earthworm. Biosci. Biotechnol. Biochem. 65, 1575-1580.

Sun, Z-J., Liang, G-D., Chen, F., Fu, S-H., Shen, Y., Chai, Y-B., Li, X-Y., Xu, Y-H. and Hou, Y-D. (2002) Lumbrukinase from Earthworm-Cloning and its effecxt to BHK cells. Chinese J. 
Biochem. Mol. Biol. 18, 776-779.

Uh, H. S., Choi, J. H., Byun, S. M., Kim, S. Y. and Lee, H. H. (2001) Cloning, sequencing and Baculovirus-based expression of fusion-glycoprotein D gene of Herpes simplex virus type 1(F). J. Biochem. Mol. Biol. 34, 371-378.
Xu, Y.-H., Liang G-D., Sun Z-J., Chem, F., Fu, S-H., Chai, Y. B. and Hou, Y-D. (2002) Cloning and expression of the novel gene-pV242 of earthworm fibrinolytic enzyme. Prog. Biochem. Biophys. 29, 610-614. 